\title{
Transport Properties of Superfluid Dipolar Fermi Gas at Low Temperatures
}

\author{
Maryam Khademi Dehkordi \\ Falavarjan Branch, Islamic Azad University, Isfahan, Iran \\ Email: khademi@iaufala.ac.ir
}

Received 2 January 2014; revised 3 March 2014; accepted 21 March 2014

Copyright (C) 2014 by author and Scientific Research Publishing Inc.

This work is licensed under the Creative Commons Attribution International License (CC BY).

http://creativecommons.org/licenses/by/4.0/

c) (i) Open Access

\section{Abstract}

Transport coefficients of superfluid dipolar Fermi gas are calculated by using the Boltzmann equation approach. The interaction between Bogoliubov-Bogoliubov quasiparticles in the collision integral is considered in binary process. The shear viscosities $\eta_{x x \uparrow}=\eta_{y y \uparrow}, \eta_{x y \uparrow} ; \eta_{x z \uparrow} \cong \eta_{y z \uparrow} ;$ and $\eta_{z z} \uparrow$ are proportional to $T^{-6} ; T^{-8}$; and $T^{-10}$, respectively. Also, we have found the elements of the diffusive thermal conductivities $K_{x x \uparrow}=K_{y y \uparrow}$ with temperature dependency $T^{-5}$ and $K_{z z}$ which is proportional to $T^{-7}$ and other components which are zero.

\section{Keywords}

Shear Viscosity, Diffusive Thermal Conductivity, Superfluid Dipolar Fermi Gas

\section{Introduction}

Fermi gases of dipolar particles present a different physical picture. Being magnetically polarized, these particles interact via anisotropic dipole-dipole forces. The potential dipole-dipole interaction is partially repulsive and partially attractive. These features of the dipole-dipole interaction have very important consequences not only for the scattering properties of the particles in ultra cold gas, but also for the stability of the system and for the variety of its properties. The recent success in observing quantum degeneracy in ultra cold atomic Fermi gas [1]-[4] stimulates a search for gaseous Fermi systems with an achievable temperature of super fluid phase transition, which is generally very low.

Fermionic superfluidity was obtained in 2003 and allowed for the exploration of BEC-BCS crossover physics, a theoretical scenario bridging the gap between the Bardeen-Cooper-Schrieffer mean-field theory describing the behavior of weakly attracting fermions (scattering length $a$ small and negative), and the strongly attractive re- 
gime ( $a$ small and positive) where the system behaves as a Bose-Einstein condensate of tightly bound dimers. Remarkably, although the theoretical foundations of this crossover physics had been laid in the early 1980s by the pioneering works of Nozières, Schmitt-Rink and Leggett [5] [6], its first experimental confirmation was only made possible by the possibility of tuning interactions in ultra-cold atom vapors using Fano-Feshbach resonances [7]-[8].

Braby et al. [9] have computed the thermal conductivity and sound attenuation length of a dilute atomic Fermi gas in the framework of kinetic theory. Above the critical temperature for superfluidity, $T_{c}$, the quasiparticles are fermions, whereas below $T_{c}$, the dominant excitations are phonons. They have calculated the thermal conductivity in both cases. They have found that at unitarity the thermal conductivity $K$ in the normal phase scales as $K \alpha T^{3 / 2}$.In the superfluid phase they have found $K \alpha T^{2}$.

The shear viscosity and the second viscosity of superfluid ${ }^{6} \mathrm{Li}$ have been formulated in the scope of Boltzmann equation approach [10] [11]. The value of the shear viscosity decreases as $\left(1-T / T_{c}\right)$ with temperature; and the second viscosity of superfluid ${ }^{6} \mathrm{Li}$ is temperature independent in the limit of low temperatures, and in the near transition temperature behaves as inverse of the gap temperature. Theoretical study show that a gas of spin-polarized atomic ${ }^{6} \mathrm{Li}$ becomes superfluid at densities and temperatures comparable with those at which the Bose-Einstein experiments are performed [12].

The shear viscosity tensor of the $A_{1}$-phase of superfluid ${ }^{3} \mathrm{He}$ is calculated at low temperatures and melting pressure, by using the Boltzmann equation approach. They obtained that the elements of the shear viscosities $\eta_{x y}, \eta_{x z}$ and $\eta_{z z}$ are proportional to $\left(T / T_{c}\right)^{-2}$ [13] [14]. Shahzamanian [15] calculated the components of the diffusive thermal conductivity of superfluid ${ }^{3} \mathrm{He}-\mathrm{A}$ using approximate collision integrals at low temperatures. The results are $K_{||} \propto T^{-4}$ and $K_{\perp} \propto T^{-2}$.

Transport coefficients of the superfluid p-wave Fermi gas with weak interaction were calculated using the Boltzmann equation approach and the quasiparticle relaxation rate [16]. They have found that the superfluid Fermi gases under their considerations are similar to ${ }^{3} \mathrm{He}-\mathrm{A}_{1}$.

Diffusive thermal conductivity tensor of p-wave superfluid was calculated using the Boltzmann equation approach at low temperatures [17]. They have shown that their results are in good agreement with $A_{1}$-phases of superfluid ${ }^{3} \mathrm{He}$ at low temperatures [18].

Here we present a calculation of the transport properties of a superfluid dipolar Fermi gas, based on the derivations of Abrikosov and Khalatnikov [19] (shear viscosity and diffusive thermal conductivity). We used a sufficiently high magnetic field so that all quasiparticles with spin up will go to superfluid state. Important collision processes are the binary scattering of quasiparticles, the decay processes in which one Bogoliubov quasi particle decays into three and coalescence processes in which three Bogoliubov quasi particles coalesce to produce one. We obtained the transition probabilities at low temperature. By using the Boltzmann equation, we calculated shear viscosity and diffusive thermal conductivity tensors.

\section{Transition Probabilities}

The interaction between quasiparticles in the superfluid dipolar Fermi gas was found by performing a Bogoliubov transformation on the normal state interaction:

$$
\begin{aligned}
& H=\frac{1}{4} \sum_{1,2,3,4}\langle 3,4|T| 1,2\rangle a_{4}^{\dagger} a_{3}^{?} a_{1} a_{2} \\
& =\frac{1}{4} \sum_{1,2,3,4}\langle 3,4|T| 1,2\rangle\left(u_{4 \uparrow \uparrow} \alpha_{4 \uparrow}^{\dagger}-v_{4 \uparrow \uparrow}^{*} \alpha_{-4 \uparrow}\right)\left(u_{3 \uparrow \uparrow} \alpha_{3 \uparrow}^{?}-v_{3 \uparrow \uparrow}^{*} \alpha_{-3 \uparrow}\right) \\
& \times\left(u_{1 \uparrow \uparrow} \alpha_{1 \uparrow}-v_{1 \uparrow \uparrow} \alpha_{-1}^{\dagger}\right)\left(u_{2 \uparrow \uparrow} \alpha_{2 \uparrow}-v_{2 \uparrow \uparrow} \alpha_{-2 \uparrow}^{?}\right) .
\end{aligned}
$$

The Hamiltonian interaction contains the terms $\alpha_{4}^{\dagger} \alpha_{3}^{?} \alpha_{-2} \alpha_{1}, \alpha_{4}^{\dagger} \alpha_{3}^{?} \alpha_{2} \alpha_{1}, \alpha_{4}^{\dagger} \alpha_{1} \alpha_{-3} \alpha_{2}, \alpha_{4}^{\dagger} \alpha_{3}^{?} \alpha_{-2} \alpha_{-1}$ and $\alpha_{-4} \alpha_{-3} \alpha_{2} \alpha_{1}$. The last two processes are not allowed because in each process the total energy should be conserved.

At low temperatures Bogoliubov coefficients can be written as $u_{p} \cong 1$ and $v_{p} \cong 0$, because more particles are gathered in the nodes of gap, that gap in direction $\boldsymbol{p}$ on the Fermi surface is $\Delta_{p}=\Delta(T) \sin \theta_{p_{\hat{r}}}$, where $\Delta(T)$ is the maximum gap and $\theta_{p}$ is the angle between the quasiparticle momentum and gap axis $\stackrel{l}{l}$, which 
is supposed to be in the direction of z-axis, in this region of temperature $\sin \theta_{p_{i}} \cong 0(i=1,2,3,4)$ [20]. Using the bogoliubov transformation, transition probabilities in polarized Fermi gas are:

$$
\begin{aligned}
& W_{22}(\uparrow \uparrow)=2 \pi|\langle 4 \uparrow 3 \uparrow|H| 1 \uparrow 2 \uparrow\rangle|^{2}=1 / 4\left|\left(V_{p_{1}-p_{3}}+V_{p_{3}-p_{1}}\right)-\left(V_{p_{3}-p_{2}}+V_{p_{2}-p_{3}}\right)\right|^{2}, \\
& W_{31}(\uparrow \uparrow)=2 \pi|\langle 4 \uparrow|H| 1 \uparrow, 2 \uparrow,-3 \uparrow\rangle|^{2} \cong 0, \\
& W_{13}(\uparrow \uparrow)=2 \pi|\langle 3 \uparrow, 4 \uparrow,-2 \uparrow|H| 1 \uparrow\rangle|^{2} \cong 0 .
\end{aligned}
$$

The interparticle interaction in polarized dipolar gases that the dipoles are polarized along the z-axis reads,

$$
V_{d}(\boldsymbol{q})=\frac{2 I^{2}}{3 \sqrt{2 \pi}}\left(3 \cos ^{2} \theta_{q}-1\right),
$$

where $\boldsymbol{I}$ is magnetic dipole, and $\theta_{q}$ is the angle between the momentum $\boldsymbol{q}$ and the z-axis [21]. Choosing $\boldsymbol{p}_{1}$ along the z-axis, $\theta_{1 i}(i=2,3,4)$ is the angle between $\boldsymbol{p}_{1}$ and $\boldsymbol{p}_{i}$, and $\varphi$ is the angle between the plane of $\left(\boldsymbol{p}_{1}, \boldsymbol{p}_{2}\right)$ with $\left(\boldsymbol{p}_{3}, \boldsymbol{p}_{4}\right)$. The relations $\cos \theta_{13}$ and $\cos \theta_{14}$ according to the momentum conservation are [22]

$$
\begin{aligned}
& \cos \theta_{13}=\cos ^{2}(\theta / 2)+\sin ^{2}(\theta / 2) \cos \varphi, \\
& \cos \theta_{14}=\cos ^{2}(\theta / 2)-\sin ^{2}(\theta / 2) \cos \varphi .
\end{aligned}
$$

At low temperatures more particles are contributing in contacts about Fermi momentum [19]. By replacing Equations (3)-(5) into (2) and considering the fact that the size of momentums are equal to Fermi momentum and by neglecting the potential effects of trap in Fermi momentum, transition probability is obtained in the form below:

$$
W(\theta, \varphi)=\frac{8 I^{4}}{\pi} \cos ^{4}(\theta / 2) \sin ^{4}(\theta / 2) \cos ^{2} \varphi
$$

\section{Collision Integral}

The Boltzmann transport equation reads

$$
\frac{\partial n}{\partial t}+\frac{\partial n}{\partial \boldsymbol{r}} \cdot \frac{\partial \varepsilon}{\partial \boldsymbol{p}}-\frac{\partial n}{\partial \boldsymbol{p}} \cdot \frac{\partial \varepsilon}{\partial \boldsymbol{r}}=I(n)
$$

where $I(n)$ is the collision integral. The full distribution function is given by $n(\boldsymbol{p}, \boldsymbol{r})=n_{0}(\boldsymbol{p})+\delta n(\boldsymbol{p})$. where $n_{0}(\boldsymbol{p})=\left(\exp \left(\varepsilon_{0}-\mu\right) / k_{B} T+1\right)^{-1}$ and $\delta n(\boldsymbol{p})$ is a small departure from equilibrium. We wrote the deviation from equilibrium as:

$$
\delta n(\boldsymbol{p})=-\frac{n_{0}(\boldsymbol{p})\left(1-n_{0}(\boldsymbol{p})\right)}{T} \chi(\boldsymbol{p}, \boldsymbol{r}),
$$

by using (8) in (7) we got [23]

$$
-\frac{1}{2} \frac{\partial n_{0}}{\partial E_{p_{0} \uparrow}} p_{i} \frac{\partial E_{p_{0} \uparrow}}{\partial p_{k}}\left(\frac{\partial u_{i}}{\partial r_{k}}+\frac{\partial u_{k}}{\partial r_{i}}-\frac{2}{3} \delta_{i j} \nabla \cdot \mathbf{u}\right)=I\left(\delta n_{\uparrow}(p)\right) .
$$

The collision integral is form [23]

$$
\begin{gathered}
I\left(\boldsymbol{p}_{1}, \uparrow\right)=\frac{\left(m^{*}\right)^{3} k_{B} T}{8 \pi^{4}} \int \mathrm{d} x \int \mathrm{d} y \int \frac{\mathrm{d} \Omega}{2 \pi} \times \int \frac{d \varphi_{2}}{2 \pi} \frac{W(\theta, \varphi)}{\cos (\theta / 2)} n_{0}(t) n_{0}(x+y-t) \\
{\left[1-n_{0}(x)\right]\left[1-n_{0}(y)\right]\left[\chi_{1}+\chi_{2}-\chi_{3}-\chi_{4}\right],}
\end{gathered}
$$

here $x=\left(\varepsilon_{3}-\mu\right) / k_{B} T, y=\left(\varepsilon_{4}-\mu\right) / k_{B} T, t=\left(\varepsilon_{1}-\mu\right) / k_{B} T$. 
We expressed $\chi(\boldsymbol{p})$ as a series of spherical harmonics in the polar angles $\Theta_{1}, \Phi_{1}$. Thus

$$
\Psi\left(\boldsymbol{p}_{1}\right)=\Psi\left(p_{1}, \Theta_{1}, \Phi_{1}\right) \sum_{n=0}^{\infty} \sum_{m=-n}^{n} \Psi_{n}^{m}(t) P_{n}^{|m|}\left(\cos \theta_{1}\right) \mathrm{e}^{\mathrm{i} m \Phi},
$$

by inserting (11) in (10) and integrate over y we have

$$
\begin{aligned}
& I_{n}^{m}(t)=\frac{\left(m^{*}\right)^{3} k_{B} T}{8 \pi^{4}} \int \mathrm{d} x \times \int \frac{\sin \theta \mathrm{d} \theta \mathrm{d} \varphi}{2 \pi} \frac{W(\theta, \varphi)}{\cos (\theta / 2)}\left(-\frac{\partial n_{0}}{\partial \varepsilon}\right) K(t, x) \\
& \times\left[\Psi_{n}^{m}(t)+\Psi_{n}^{m}(-x) \times P_{n}(\cos \theta)-\Psi_{n}^{m}(x)\left\{P_{n}\left(\cos \theta_{3}\right)+P_{n}\left(\cos \theta_{4}\right)\right\}\right],
\end{aligned}
$$

where

$$
K(t, x)=\frac{\mathrm{e}^{-t}+1}{\mathrm{e}^{-x}+1} \frac{(x-t)}{\mathrm{e}^{(x-t)}-1} .
$$

We divided $\Psi_{n}^{m}(t)$ in to even and odd functions of $t$, and in the same way separated $I_{n}^{m}(t)$. Since $K(t, x)=K(-t,-x)$, only the even part of $\Psi_{n}^{m}(t)$ contributes to the even part of $I$, and similarly for the odd part [24].

$$
\begin{gathered}
\int \mathrm{d} x K(t, x)\left\{\Psi_{n s}^{m}(t)-\lambda_{n s} \Psi_{n s}^{m}(x)\right\}=\text { an even function of } t, \\
\int \mathrm{d} x K(t, x)\left\{\Psi_{n s}^{m}(t)-\lambda_{n s} \Psi_{n s}^{m}(x)\right\}=\text { an odd function of } t,
\end{gathered}
$$

that an even function of $t$ used for calculating of shear viscosity and odd function used for thermal conductivity [24].

\section{Shear Viscosity}

The coefficients shear viscosity describes the response of the momentum current density to a transverse velocity field

$$
\Pi_{i j}=-\sum_{i j k l} \eta_{i j k l}\left(\frac{\partial u_{i}}{\partial r_{k}}+\frac{\partial u_{k}}{\partial r_{i}}-\frac{2}{3} \delta_{i j} \nabla \cdot u\right),
$$

$\Pi_{i j}$ is expressed in terms of the bogoliubov qusipartcle distribution function $\delta n_{p}$ by

$$
\Pi_{i j}=\sum_{p} p_{i}\left(n_{p}\right)_{j} \delta n_{p}^{\prime}
$$

to solve the linearized Boltzmann equation, it is suitable to define $q(t)$ as

$$
\chi(t)=p_{i} \frac{\partial E_{1}}{\partial p_{1 k}} q(t)\left[\frac{\partial u_{i}}{\partial r_{k}}+\frac{\partial u_{k}}{\partial r_{i}}-\frac{2}{3} \delta_{i j} \nabla \cdot u\right] .
$$

By substituting (18) in (9) and then in (17) and compared with (16), we got [24]

$$
\eta_{i j k l}=-\frac{4 p_{f}^{5}}{(2 \pi)^{3} m^{*}} \int \mathrm{d} \Omega_{p} \hat{p}_{i} \hat{p}_{j} \hat{p}_{k} \hat{p}_{l}\left(\int \mathrm{d} t \frac{\partial n_{0}}{\partial t} q_{2 s \uparrow}(t)\right) .
$$

By solving the even part of $t$ in (15) and with regard to the fact that system is polarized, we have [24],

$$
\eta_{i j k l}=\frac{p_{f}^{5}}{\pi^{5} m^{*}} \int \mathrm{d} \Omega_{p} \hat{p}_{i} \hat{p}_{j} \hat{p}_{k} \hat{p}_{l}\left(\frac{B_{\uparrow}}{1-\lambda_{2 s \uparrow}} C\left(\lambda_{2 s \uparrow}\right)\right),
$$

in this equation $B_{\uparrow}, \quad \lambda_{2 s \uparrow}$ and $C\left(\lambda_{2 s \uparrow}\right)$ are

$$
B_{\uparrow}=\frac{16 \pi^{5}}{\left(m^{*}\right)^{3} T^{2}}\left[\int \frac{\sin \theta \mathrm{d} \theta \mathrm{d} \varphi}{\cos (\theta / 2)} W(\theta, \varphi)\right]^{-1},
$$




$$
\begin{array}{r}
\lambda_{2 s \uparrow}=\frac{\int \frac{\sin \theta \mathrm{d} \theta \mathrm{d} \varphi}{\cos (\theta / 2)} W(\theta, \varphi)\left\{1-\frac{3}{4}(1-\cos \theta)^{2} \sin ^{2} \varphi\right\}}{\int \frac{\sin \theta \mathrm{d} \theta \mathrm{d} \varphi}{\cos (\theta / 2)} W(\theta, \varphi)}, \\
C\left(\lambda_{2 s \uparrow}\right)=\frac{1-\lambda_{2 s \uparrow}}{4} \sum_{n=0}^{\infty} \frac{4 n+3}{(n+1)(2 n+1)\left\{(n+1)(2 n+1)-\lambda_{2 s \uparrow}\right\}} \\
=\frac{\lambda_{2 s \uparrow}-1}{2 \lambda_{2 s \uparrow}}\left\{\gamma+\ln 2+\frac{1}{2} \psi_{d}\left(s_{1}\right)+\frac{1}{2} \psi_{d}\left(s_{2}\right)\right\},
\end{array}
$$

and $\gamma=0.577 \cdots$ is Euler's constant, $\psi_{d}$ is a digamma function and

$$
s_{1}=\frac{3}{4}+\frac{1}{4} \sqrt{8 \lambda+1}, \quad s_{2}=\frac{3}{4}-\frac{1}{4} \sqrt{8 \lambda+1} .
$$

$W(\theta, \varphi)$ only replaces with $\frac{1}{4} W_{22}(\uparrow \uparrow)$, because $W_{13}(\uparrow \uparrow)$ and $W_{31}(\uparrow \uparrow)$ are nearly zero.

For obtaining $\lambda_{2 s \uparrow}$ and $B_{\uparrow}$, we used (6) and note that $\theta$ is small for superfluid case and its maximum value is $\pi T / \Delta(0)$ [20], where maximum gap, $\Delta(0)$, due to strong coupling effects is equal to $1.76 T_{c}$ [25]. Then, we obtained:

$$
\begin{gathered}
\lambda_{2 s \uparrow}=1-\frac{9}{320} \theta_{m}^{4}, \\
B_{\uparrow}=\frac{192 \pi^{5}}{\left(m^{*}\right)^{3} k_{B}^{2} T^{2} I^{4} \theta_{m}^{6}}, \\
C\left(\lambda_{2 s \uparrow}\right)=\left[0.75+0.003675 \theta_{m}^{4}\right] .
\end{gathered}
$$

After substituting $B_{\uparrow}, \lambda_{2 \uparrow}$, and $C\left(\lambda_{2 \uparrow}\right)$ in (20) and taking the angular integrations, we have

$$
\begin{gathered}
\eta_{x x \uparrow}=\eta_{y y \uparrow}=1943 \cdot 237 \frac{p_{f}^{5}}{\left(m^{*}\right)^{4} k_{B}^{2} I^{4}} \frac{T_{c}^{4}}{T^{6}}, \\
\eta_{z z \uparrow}=156 \cdot 62 \frac{p_{f}^{5}}{\left(m^{*}\right)^{4} k_{B}^{2} I^{4}} \frac{T_{c}^{8}}{T^{10}}, \\
\eta_{x y \uparrow}=3 \cdot 503 \times 10^{-6} \frac{p_{f}^{5}}{\left(m^{*}\right)^{4} k_{B}^{2} I^{4}} \frac{T_{c}^{4}}{T^{6}}, \\
\eta_{x z \uparrow}=123 \cdot 84 \frac{p_{f}^{5}}{\left(m^{*}\right)^{4} k_{B}^{2} I^{4}} \frac{T_{c}^{6}}{T^{8}}, \\
\eta_{y z \uparrow}=124 \cdot 63 \times 10^{-6} \frac{p_{f}^{5}}{\left(m^{*}\right)^{4} k_{B}^{2} I^{4}} \frac{T_{c}^{6}}{T^{8}} .
\end{gathered}
$$

\section{Thermal Conductivity}

The diffusive thermal conductivity tensor $K_{i j}$ is defined by

$$
J_{i}=K_{i j} \nabla_{j} T,
$$


where $\boldsymbol{J}$ is the diffusive heat current. It is noted that, in addition to heat transfer by a random diffusive process of thermal excitations described by Equations (33), there is a convective contribution to the heat current in a superfluid, even in the absence of mass flow, owing to the possibility of normal-superfluid counter flow.

At low temperatures, this contribution is negligible [24]-[26] and the measurement of the components of thermal conductivity at these temperatures is more meaningful than the measurement of the thermal conductivity components in the vicinity of $T_{c}$.

The diffusive heat current may be written in terms of the polarized quasi-particle distribution functions:

$$
J_{i}=\int \mathrm{d} \tau E \frac{\partial E}{\partial p_{i}} \delta n_{p \uparrow},
$$

$\delta n_{p \uparrow}$ is defined at below[26]

$$
\delta n_{p \uparrow}=\frac{-n_{0}(p)\left(1+n_{0}(p)\right)}{T}\left(\frac{\partial E_{1}}{\partial p_{1 k}}\right) q(t)\left[\frac{\partial T}{\partial r_{k}}\right] .
$$
[24]

By using (35) in (34) and compared with (33) and using odd function of $t$ in (15) for polarized gas we got

$$
K_{i j}=-\frac{4 p_{f}^{3}}{\left(m^{*}\right)^{3}(2 \pi)^{3}} T \int \mathrm{d} \Omega_{p} \hat{p}_{i} \hat{p}_{j}\left(\int \mathrm{d} t \frac{\partial n_{0 \uparrow}}{\partial t} q_{1 a \uparrow}(t) t\right) .
$$

By using Sykes and Brooker procedure we defined $K_{i j}$ as bellows

$$
K_{i j}=\frac{8 p_{f}^{3} T}{\left(m^{*}\right)^{3}(2 \pi)^{3}} T \int \mathrm{d} \Omega_{p} \hat{p}_{i} \hat{p}_{j}\left(\frac{B_{1 a \uparrow}}{3-\lambda_{1 a \uparrow}} H\left(\lambda_{1 \uparrow \uparrow}\right)\right),
$$

$\lambda_{1 a \uparrow}$ and $H\left(\lambda_{1 a \uparrow}\right)$ are introduced as below

$$
\begin{array}{r}
\lambda_{1 a \uparrow}=\frac{\int \frac{\sin \theta \mathrm{d} \theta d \varphi}{\cos (\theta / 2)} W(\theta, \varphi)(1+2 \cos \theta)}{\int \frac{\sin \theta \mathrm{d} \theta \mathrm{d} \varphi}{\cos (\theta / 2)} W(\theta, \varphi)}, \\
H(\lambda)=\frac{3-\lambda_{1 a \uparrow}}{4} \sum_{n=0}^{\infty} \frac{(4 n+5)}{(n+1)(2 n+3)\left\{(n+1)(2 n+3)-\lambda_{1 a \uparrow}\right\}} \\
=\frac{\lambda_{1 a \uparrow}-3}{2 \lambda_{1 a \uparrow}}\left\{\gamma+\ln 2-1-\frac{1}{2 \lambda_{1 a \uparrow}}+\frac{1}{2} \psi_{d}\left(s_{1}-\frac{1}{2}\right)+\frac{1}{2} \psi_{d}\left(s_{2}-\frac{1}{2}\right)\right\},
\end{array}
$$

where $s_{1}$ and $s_{2}$ are defined by (24).

By using (6) in (38), (39) we took the following explicit forms

$$
\begin{gathered}
\lambda_{1 a \uparrow}=1-\frac{3}{4} \theta_{m}^{2}, \\
H\left(\lambda_{1 a \uparrow}\right)=0.42-0.595 \theta_{m}^{2} .
\end{gathered}
$$

By using the Sykes and Brooker procedure [24], we calculated the diffiusive thermal conductivity tensor. After substituting $\lambda_{1 a \uparrow}, B_{\uparrow}$, and $H\left(\lambda_{1 a \uparrow}\right)$ in (37), and taking the angular integrations, we got:

$$
\begin{gathered}
K_{x y \uparrow}=K_{x z \uparrow}=K_{y z \uparrow}=0, \\
K_{x x \uparrow}=K_{y y \uparrow}=97.78 \frac{p_{f}^{3} T_{c}^{4}}{\left(m^{*}\right)^{6} k_{B}^{2} I^{4} T^{5}},
\end{gathered}
$$




$$
K_{z z} \uparrow=122.88 \frac{p_{f}^{3} T_{c}^{6}}{\left(m^{*}\right)^{6} k_{B}^{2} I^{4} T^{7}} .
$$

\section{Discussion and Concluding Remarks}

As a result, in a superfluid dipolar Fermi gases we calculated the transition probabilities for the cases where the Bogoliubov quasiparticles are presented in coalescence, decay and binary processes and obtained that binary processes are dominated in calculation of transition probabilities, and other processes are nearly zero. Then, by using collision integral in the Boltzmann equation contained binary processes, the components of shear viscosity and thermal conductivity were calculated. We obtained $\eta_{x x \uparrow}=\eta_{y y \uparrow}, \eta_{x y \uparrow} ; \eta_{x z \uparrow} \cong \eta_{y z \uparrow}$; and $\eta_{z z \uparrow}$ with temperatures dependences $T^{-6} ; T^{-8}$; and $T^{-10}$ respectively (see Equations (28)-(32)); and we have found the components of the diffusive thermal conductivity, $K_{x x \uparrow}=K_{y y \uparrow}$ with temperature dependency $T^{-5}$ and $K_{z z} \uparrow$ which is proportional to $T^{-7}$ and other components which are zero. Temperature dependency of shear viscosity and thermal conductivity along the z-axis ( $T^{-10}$ and $T^{-7}$ respectively) were showing that the polarization is along the z-axis.

Then, we had to compare our results in transport coefficients with [13] [14]. (They found the shear viscosities $\eta_{x y}, \eta_{x z}$ and $\eta_{z z}$ of $\mathrm{A}_{1}$-phase of superfluid ${ }^{3} \mathrm{He}$ are proportional to $\left(\mathrm{T} / \mathrm{T}_{\mathrm{c}}\right)^{-2}$.). Two major differences were shown: a) Transition probabilities in superfluid dipolar Fermi gas are the function of temperature, but in superfluid ${ }^{3} \mathrm{He}-\mathrm{A}_{1}$ are constant. b) ${ }^{3} \mathrm{He}-\mathrm{A}_{1}$ have two kind of the quasiparticles, one for Bogoliubov quasiparticle, and another for normal quasiparticle. The wave function of the system in $A_{1}$ phases of ${ }^{3} \mathrm{He}$ is $|\uparrow \uparrow\rangle$ that interacts with Bogoliubov quasipaticle $(\uparrow)$ and normal quasiparticle $(\downarrow)$, but in superfluid dipolar Fermi gas only $|\uparrow \uparrow\rangle$ contributes to Bogoliubov quasiparticles $(\uparrow)$ in interaction.

It is noted that, in superfluid Fermi gas in p-wave state $W_{22}(\uparrow \uparrow), W_{22}(\uparrow \downarrow)$ were contributed to calculations [16] [17] but in superfluid dipolar Fermi gas only $W_{22}(\uparrow \uparrow)$ with temperature dependency contributes to calculations.

Also, in [15] the components of thermal conductivity tensor of superfluid ${ }^{3} \mathrm{He}-\mathrm{A}$ were calculated using approximate collision integrals at low temperatures, and the thermal coefficients $K_{\perp}$ and $K_{\|}$have been obtained with temperature dependences $T^{-1}$ and $T$, respectively. This is due to the fact that $\lambda_{1}^{-}$ $\left(\lambda_{1}^{-}=1+(2 /\langle W\rangle)\langle W(\theta, \varphi) \cos \theta\rangle\right)$ was taken to be a constant parameter with respect to temperatures.

In [18], $W_{22}(\uparrow \uparrow), W_{22}(\downarrow \downarrow)$ and $W_{22}(\uparrow \downarrow)$ were contributed in calculations and the wave function $|\uparrow \uparrow\rangle$ interacted with Bogoliubov and normal quasiparticles, but in superfluid dipolar gas only $W_{22}(\uparrow \uparrow)$ contributed to calculation and only $|\uparrow \uparrow\rangle$ interacted with Bogoliubov quasiparticles. These differences in transition probabilities of ${ }^{3} \mathrm{He}-A_{1}$ and superfluid Fermi gas in p-wave state with superfluid dipolar Fermi gas, have shown themselves in transport coefficients.

\section{References}

[1] deMarco, B. and Jin, D.S. (1999) Onset of Fermi Degeneracy in a Trapped Atomic Gas. Science, 285, 1703-1706. http://dx.doi.org/10.1126/science.285.5434.1703

[2] deMarco, B., Papp, S.B. and Jin, D.S. (2001) Pauli Blocking of Collisions in a Quantum Degenerate Atomic Fermi Gas. Physical Review Letters, 86, Article ID: 5409. http://dx.doi.org/10.1103/PhysRevLett.86.5409

[3] Truscott, A.G., Strecker, K.E., McAlexander, W.I., Partridge, G.B. and Hulet, R.G. (2001) Observation of Fermi Pressure in a Gas of Trapped Atoms. Science, 291, 2570-2572. http://dx.doi.org/10.1126/science.1059318

[4] Schreck, F., Khaykovich, L., Corwin, K.L., Ferrari, G., Bourdwel, T., Cubizolles, J. and Salomon, C. (2001) Quasipure Bose-Einstein Condensate Immersed in a Fermi Sea. Physical Review Letters, 87, Article ID: 080403. http://dx.doi.org/10.1103/PhysRevLett.87.080403

[5] Leggett, A.J. (1980) Cooper Pairing in Spin-Polarized Fermi Systems. Journal de Physique Archives, 41, 19-26. http://dx.doi.org/10.1051/jphyscol:1980704

[6] Nozières, P. and Schmitt-Rink, S. (1985) Bose Condensation in an Attractive Fermion Gas: From Weak to Strong Coupling Superconductivity. Journal of Low Temperature Physics, 59, 195-211. http://dx.doi.org/10.1007/BF00683774

[7] Jochim, S., Bartenstein, M., Altmeyer, A., Hendl, G., Riedl, S., Chin, C., Denschlag, J.H. and Grimm, R. (2003) BoseEinstein Condensation of Molecules. Science, 302, 2101-2103. 
[8] Bourdel, T., Khaykovich, L., Cubizolles, J., Zhang, J., Chevy, F., Teichmann, M., Tarruell, L., Kokkelmans, S.J.J.M.F. and Salomon, C. (2004) Experimental Study of the BEC-BCS Crossover Region in Lithium 6. Physical Review Letters, 93, Article ID: 050401. http://dx.doi.org/10.1103/PhysRevLett.93.050401

[9] Braby, M., Jingyi, C. and Schäfer, T. (2010) Thermal Conductivity and Sound Attenuation in Dilute Atomic Fermi Gases. Physical Review A, 82, Article ID: 033619. http://dx.doi.org/10.1103/PhysRevA.82.033619

[10] Shahzamanian, M.A. and Yavary, H. (2002) The Shear Viscosity of Superfluid ${ }^{6}$ Li. Physica B, 321, 385-387. http://dx.doi.org/10.1016/S0921-4526(02)01080-3

[11] Shahzamanian, M.A. and Yavary, H. (2002) The Second Viscosity of Superfluid ${ }^{6}$ Li. International Journal of Modern Physics B, 16, 3797-3802. http://dx.doi.org/10.1142/S0217979202013092

[12] Stoof, H.T.C., Houbiers, M., Sacket, C.A. and Hulet, R.G. (1996) Superfluidity of Spin-Polarized ${ }^{6}$ Li. Physical Review Letters, 76, Article ID: 10. http://dx.doi.org/10.1103/PhysRevLett.76.10

[13] Shahzamanian, M.A. and Afzali, R. (2004) Shear Viscosity of Superfluid ${ }^{3} \mathrm{He}-\mathrm{A}_{1}$ at Low Temperature. Annals of Physics, 309, 281-294. http://dx.doi.org/10.1016/j.aop.2003.08.012

[14] Shahzamanian, M.A. and Afzali R. (2004) Quasiparticle Relaxation Rate and Shear Viscosity of Superfluid ${ }^{3} \mathrm{He}^{-\mathrm{A}_{1}}$ at Low Temperatures. Physica B, 348, 108-114. http://dx.doi.org/10.1016/j.physb.2003.11.078

[15] Shahzamanian, M.A. (1989) Diffusive Thermal Conductivity of Superfluid ${ }^{3}$ He-A1 at Low Temperatures. Journal of Physics: Condensed Matter, 1, 1965-1970. http://dx.doi.org/10.1088/0953-8984/1/11/003

[16] Nasirimoghadam, S., Nabipoor, F., Khademi Dehkordi, M. and Shahzamanian, M.A. (2012) Transport Coefficients of the Superfluid Fermi gas in P-Wave State at Low Temperature. Physica C: Superconductivity, 483, 109-112. http://dx.doi.org/10.1016/j.physc.2012.05.020

[17] Khademi Dehkordi, M., Nasirimoghadam, S., Nabipoor, F. and Shahzamanian, M.A. (2012) Diffusive Thermal Conductivity of p-Wave State at Low Temperatures. Communications in Theoretical Physics, 58, 891-894. http://dx.doi.org/10.1088/0253-6102/58/6/16

[18] Afzali, R. and Ebrahimian, N. (2005) Diffusive Thermal Conductivity of the $\mathrm{A}_{1}$-Phase of Superfluid ${ }^{3} \mathrm{He}$ at Low Temperatures. Journal of Physics: Condensed Matter, 17, 4441. http://dx.doi.org/10.1088/0953-8984/17/28/004

[19] Abrikosov, A.A. and Khalatnikov, I.M. (1959) The Theory of a Fermi Liquid. Reports on Progress in Physics, $22,329$. http://dx.doi.org/10.1088/0034-4885/22/1/310

[20] Takagi, S. (1975) On the NMR Properties near the ${ }^{3}$ He A-Normal Transition. Journal of Low Temperature Physics, 18, 309-314. http://dx.doi.org/10.1007/BF00118160

[21] Baranov, M.A. (2008) Theoretical Progress in Many-Body Physics with Ultracold Dipolar Gases. Physics Reports, 464, 71-111. http://dx.doi.org/10.1016/j.physrep.2008.04.007

[22] Pethick, C.J., Smith, H. and Bhattacharyya, P. (1977) Transport Processes in Superfluid ${ }^{3}$ He-B at Low Temperatures. Physical Review B, 15, 3384. http://dx.doi.org/10.1103/PhysRevB.15.3384

[23] Volhardt, D. and Wolfle, P. (1990) The Superfluid Phases of Helium 3. Taylor \& Francis, London.

[24] Sykes, J. and Brooker, G.A. (1970) The Transport Coefficients of a Fermi Liquid. Annals of Physics, 56, 1-39. http://dx.doi.org/10.1016/0003-4916(70)90002-3

[25] Pethick, C.J. and Smith, H. (2002) Bose-Einestein Condensation in Dilute Gases. Cambridge University Press, Cambridge.

[26] Wolfle, P. and Einzel, D. (1978) Transport and Relaxation Properties of Superfluid ${ }^{3}$ He. Journal of Low Temperature Physics, 32, 39-56. http://dx.doi.org/10.1007/BF00116905 